\title{
OCORRÊNCIA E PERFIL DE SENSIBILIDADE A ANTIBIÓTICOS DE Salmonella spp. ISOLADA EM CORTES DE CARNE SUÍNA COMERCIALIZADOS EM FEIRAS-LIVRES DE PELOTAS (RS)
}

\author{
CRISTIANE TESSMANN* \\ FERNANDO ZOCCHE** \\ ANDRÉIA SALDANHA DE LIMA*** \\ MILENA BASSANI ${ }^{\star \star \star \star *}$

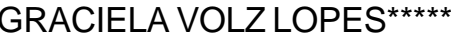 \\ WLADIMIR PADILHADA SILVA ${ }^{\star \star \star \star \star \star ~}$
}

\begin{abstract}
O objetivo deste estudo foi avaliar a ocorrência de Salmonella spp. em 15 amostras de cortes de carne suína (chuleta, pernil, copa e costela), comercializados em feiras-livres na cidade PelotasRS, verificar os sorovares prevalentes, e testar os isolados frente a diversos antibióticos de importância em clínica médica (ácido nalidíxico, ampicilina, aztreonam, canamicina, carbenicilina, cefalotina, cefoxitina, ceftriaxona, ciprofloxacina, cloranfenicol, gentamicina, sulfonamida, tetraciclina e trimetoprina). Doze amostras (80\%) apresentaram contaminação por Salmonella enterica, sorovares Infantis, Derby, Panama e Typhimurium. Todos os isolados foram sensíveis à trimetoprina, aztreonam, ciprofloxacina, ceftriaxona e cefoxitina. Quanto aos demais antibióticos, o padrão de sensibilidade variou conforme o sorovar. Além disso, 39,1\% dos isolados apresentaram-se multirresistentes.
\end{abstract}

PALAVRAS-CHAVE: CONTAMINAÇÃO MICROBIOLÓGICA; ANTIBIÓTICOS, CARNE SUÍNA; Salmonella; MULTIRRESISTEENCIA.

\footnotetext{
* Bióloga, M.Sc. em Ciências, Centro Federal de Educação Tecnológica da Bahia (CEFET), Porto Seguro, BA (e-mail: cbkika@yahoo.com.br).

** Médico Veterinário, Doutor em Ciências, Departamento de Ciência e Tecnologia Agroindustrial (DCTA), Faculdade de Agronomia Eliseu Maciel (FAEM), Universidade Federal de Pelotas (UFPel), Pelotas, RS, (e-mail: fernandozocche@hotmail.com).

*** Química de Alimentos, M.Sc. em Ciências, DCTA, FAEM, UFPel, Pelotas, RS (e-mail: andreia@ufpel. edu.br).

**** Médica Veterinária, DCTA, FAEM, UFPel, Pelotas, RS (e-mail: mtbassani@yahoo.com.br).

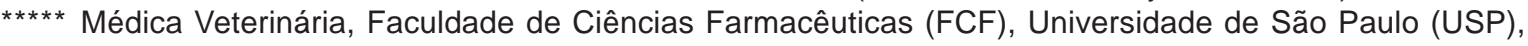
São Paulo, SP (e-mail: gracielavlopes@yahoo.com.br).

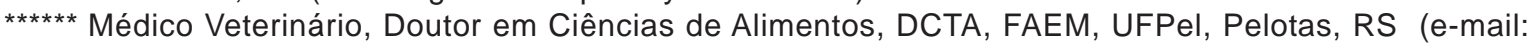
silvawp@ufpel.edu.br)
} 


\section{INTRODUÇÃO}

A salmonelose constitui doença de grande importância econômica e em saúde pública, sendo a principal doença transmitida por alimentos (DTA) no sul do Brasil (COSTALUNGA e TONDO, 2002). Ovos e carne de aves são os alimentos mais envolvidos nos surtos de salmonelose em humanos, entretanto estima-se que a carne suína e seus derivados sejam responsáveis por 15-20\% dos casos (BERENDS et al., 1996).

Muitos sorovares de Salmonella têm como reservatório determinada espécie animal, estando sua transmissão associada com alimentos obtidos a partir desses animais (BAÚ, CARVALHAL e ALEIXO, 2001). Tratando-se microrganismo de origem intestinal, animais saudáveis são freqüentemente infectados sem apresentar qualquer sintoma da doença. Os suínos, por exemplo, são reservatórios de diversos sorovares de Salmonella enterica, assim a carne suína e seus derivados têm sido associados com a veiculação dessa bactéria pelos alimentos (CASTAGNA et al., 2004; LIMA et al., 2004; LOGUÉRCIO et al., 2002). Nesse aspecto, BERSOT (2004), em revisão sobre a cadeia produtiva de suínos e disseminação de Salmonella relata que o isolamento desse patógeno é freqüente ao longo da cadeia produtiva e que Salmonella pode estar presente nas etapas de produção, transporte, descanso pré-abate e abate de suínos. A presença desse microrganismo torna-se grande problema pelo fato das carcaças contaminadas com bactérias patogênicas não serem identificadas pela inspeção veterinária durante o abate (SWANENBURG et al., 2001).

O uso indiscriminado (ou incorreto) de antibióticos na medicina humana, na veterinária e na agricultura, tem contribuído muito para o aumento de resistência dos microrganismos a esses agentes (WHO, 2000), que pode ocorrer pela transferência vertical ou horizontal de genes de resistência (WITTE, 2004). Aemergência de organismos resistentes a múltiplos antibióticos, incluindo Salmonella (FLUIT, 2005), constitui problema crescente para a saúde pública (KHAN, MELVIN e DAGGAG, 2007). A determinação do perfil de sensibilidade e resistência a antimicrobianos entre os sorovares de salmonelas isolados de alimentos em determinada região, além de ser importante como marcador epidemiológico, serve para orientar procedimentos terapêuticos em medicina humana e veterinária (BAÚ, CARVALHAL e ALEIXO, 2001).

Em várias cidades do Brasil, incluindo Pelotas (RS), são comuns feiras-livres que comercializam gêneros alimentícios de origem vegetal e animal. Embora a legislação brasileira estabeleça obrigatoriedade da inspeção oficial para comercialização de alimentos de origem animal, nem sempre os produtos vendidos em feiras-livres são procedentes de estabelecimentos fiscalizados. Nesse caso, tais produtos são enquadrados como clandestinos.

O objetivo deste estudo foi avaliar a ocorrência de Salmonella spp. em cortes de carne suína comercializados em feiras-livres na cidade Pelotas-RS, verificar os sorovares prevalentes, e testar os isolados frente a diversos antibióticos de importância em clínica médica.

\section{MATERIAL E MÉTODOS}

\subsection{AMOSTRAS DE PRODUTOS DERIVADOS DE CARNE SUÍNA}

Quinze amostras de cortes de carne suína (chuleta, pernil, copa e costela) foram obtidas em feiras-livres de Pelotas (RS), no período de junho a agosto de 2006. As amostras foram coletadas nas embalagens utilizadas pelo comerciante, acondicionadas em caixas de isopor contendo gelo, e transportadas ao laboratório de Microbiologia de Alimentos da Universidade Federal de Pelotas (UFPel), sendo imediatamente submetidas às análises microbiológicas.

\subsection{ISOLAMENTO DE Salmonella spp.}

Utilizou-se para o isolamento de Salmonella spp. o método preconizado pela APHA (2001). As amostras foram homogeneizadas em stomacher (ITR Ltda ${ }^{\circledR}$ ), usando-se $25 \mathrm{~g}$ de amostra em 
$225 \mathrm{~mL}$ de água peptonada tamponada (APT, Merck ${ }^{\circledR}$ ) para incubação a $37^{\circ} \mathrm{C}$ durante $24 \mathrm{~h}$. Após esse período, transferiu-se $1 \mathrm{~mL}$ para $10 \mathrm{~mL}$ de caldo Tetrationato (TT, Acumedia ${ }^{\circledR}$ ) e $0,1 \mathrm{~mL}$ para $10 \mathrm{~mL}$ de caldo Rappaport-Vassiliadis (RV, Oxoid ${ }^{\circledR}$ ). O caldo TT foi incubado a $37^{\circ} \mathrm{C} / 24 \mathrm{~h}$ e o caldo RV a $42^{\circ} \mathrm{C} / 24 \mathrm{~h}$. Após o período de incubação, uma alçada proveniente de cada caldo de enriquecimento seletivo foi estriada em placas de Petri contendo ágar Entérico de Hectoen (HE, Acumedia ${ }^{\circledR}$ ), em ágar Xilose Lisina Desoxicolato (XLD, Acumedia ${ }^{\circledR}$ ) e em ágar Salmonella Shigella (SS, Himedia ${ }^{\circledR}$ ), as quais foram incubadas a $37^{\circ} \mathrm{C} /$ 24h. O plaqueamento seletivo diferencial também foi realizado em ágar Bismuto de Sulfito (BS, Acumedia ${ }^{\circledR}$ ), o qual foi incubado a $37^{\circ} \mathrm{C} / 48 \mathrm{~h}$. Pelo menos duas colônias em cada placa, suspeitas de serem salmonelas, foram submetidas à identificação bioquímica mediante ágar Tríplice Açúcar e Ferro (TSI, Acumedia ${ }^{\circledR}$ ), ágar Lisina e Ferro (LIA, Micromed ${ }^{\circledR}$ ) e ágar Uréia (UA, Vetec $\left.{ }^{\circledR}\right)$. Os isolados que apresentaram comportamento bioquímico característico do gênero Salmonella foram submetidos à prova de soroaglutinação rápida em lâmina, empregando-se soro polivalente somático e flagelar (Probac do Brasi $\left.^{\circledR}\right)$. A identificação dos isolados em nível de sorotipo foi realizada pelo Laboratório de Enterobactérias, Departamento de Bacteriologia, da Fundação Oswaldo Cruz, Rio de Janeiro.

\subsection{AVALIAÇÃO DA SENSIBILIDADE AANTIBIÓTICOS}

Realizou-se a avaliação da sensibilidade a antibióticos pelo método de disco-difusão de BAUER e KIRBY (1966), interpretado de acordo com o NCCLS (1984). Discos de papel filtro, impregnados com os agentes antimicrobianos, foram colocados em placas de petri contendo meio Mueller-Hinton (Merck ${ }^{\circledR}$ ) inoculado com os isolados a serem testados, numa densidade equivalente ao padrão 0,5 da Escala de MacFarland $\left(1,6 \times 10^{8}\right.$ bactérias $\left.\mathrm{mL}^{-1}\right)$. Testou-se a sensibilidade dos isolados frente ao ácido nalidíxico $(30 \mu \mathrm{g})$, ampicilina $(10 \mu \mathrm{g})$, aztreonam $(30 \mu \mathrm{g})$, canamicina $(30 \mu \mathrm{g})$, carbenicilina $(100 \mu \mathrm{g})$, cefalotina $(30 \mu \mathrm{g})$, cefoxitina $(30 \mu \mathrm{g})$, ceftriaxona $(30 \mu \mathrm{g})$, ciprofloxacina $(5 \mu \mathrm{g})$, cloranfenicol $(30 \mu \mathrm{g})$, gentamicina $(10 \mu \mathrm{g})$, sulfonamida $(300 \mu \mathrm{g})$, tetraciclina $(30 \mu \mathrm{g})$ e trimetoprina $(5 \mu \mathrm{g})$.

\section{RESULTADOS E DISCUSSÃO}

Do total de amostras analisadas $(n=15), 12(80 \%)$ estavam contaminadas com Salmonella spp. (Tabela 1) e portanto em desacordo com a Resolução 12, de 02 de janeiro de 2001 (BRASIL, 2001).

\section{TABELA 1 - ISOLAMENTO DE Salmonella spp. A PARTIR DE CORTES DE CARNE SUÍNA COMERCIALIZADOS EM FEIRAS-LIVRES NA CIDADE DE PELOTAS, RS}

\begin{tabular}{cccccc}
\hline Amostras $(\mathrm{n}=15)$ & Chuleta $\mathrm{n}(\%)$ & Pernil $\mathrm{n}(\%)$ & Costela $\mathrm{n}(\%)$ & Copa $\mathrm{n}(\%)$ & Total $\mathrm{n}(\%)$ \\
\hline Presença de & $5(83,3)$ & $3(60)$ & $3(100)$ & $1(100)$ & $12(80)$ \\
Salmonella spp. & & & & & $3(20)$ \\
Ausência de & $1(16,7)$ & $2(40)$ & - & - & $15(100)$ \\
$\begin{array}{c}\text { Salmonella spp. } \\
\text { Total }\end{array}$ & $6(100)$ & $5(100)$ & $3(100)$ & $1(100)$ & 15 \\
\hline
\end{tabular}

A ocorrência de Salmonella spp. em $80 \%$ das amostras analisadas é preocupante e ressalta a importância do consumo de alimentos com procedência sanitária. Também demonstra a relevância desse tipo de produto na veiculação de casos e/ou surtos de salmonelose, seja pelo seu consumo ou mediante contaminação cruzada para outros alimentos.

Não foi possível estabelecer a origem das carnes avaliadas e dessa forma não se sabe se foram ou não submetidas à inspeção veterinária oficial. De qualquer maneira, o nível de contaminação encontrado demonstra a falta de cuidado na sua manipulação durante o abate e/ou comercialização. É importante ressaltar que outros estudos conduzidos na região de Pelotas, em nível de frigorífico, 
demonstraram o mesmo tipo de contaminação de carcaças, de carne e gordura suínas utilizadas como matéria-prima para embutidos, e de cortes de pernil (DUVAL et al., 2003, BANDEIRA et al., 2003).

Já foram descritos mais de 2300 sorovares de Salmonella e embora todos sejam considerados patogênicos para humanos, alguns fatores de virulência parecem estar presentes em limitado número deles (FLUIT, 2005). Dessa forma, a sorotipagem e a avaliação da resistência a antibióticos constituem importantes ferramentas, tanto para investigações epidemiológicas, quanto para o estabelecimento de estratégias terapêuticas de uso de antibióticos. Todos os isolados pertenciam a espécie $S$. enterica, sendo identificados 4 sorovares: Infantis, Typhimurium, Panama e Derby. Aocorrência dos diferentes sorovares nas amostras está descrita na Tabela 2, podendo-se observar que houve prevalência do sorovar Infantis.

\section{TABELA 2 - DISTRIBUIÇÃO DE SOROVARES DE Salmonella spp. EM CORTES DE CARNE SUÍNA COMERCIALIZADOS EM FEIRAS-LIVRES NA CIDADE DE PELOTAS, RS}

\begin{tabular}{ccccc}
\hline Sorovar & Chuleta $\mathrm{n}(\%)$ & Pernil $\mathrm{n}(\%)$ & Costela $\mathrm{n}(\%)$ & Copa n (\%) \\
\hline Infantis & $4(57,1)$ & $3(37,5)$ & $6(100)$ & $1(50)$ \\
Typhimurium & $2(28,6)$ & $4(50)$ & $0(0)$ & $1(50)$ \\
Panama & $1(14,3)$ & $0(0)$ & $0(0)$ & $0(0)$ \\
Derby & $0(0)$ & $1(12,5)$ & $0(0)$ & $0(0)$ \\
Total & $7(100)$ & $8(100)$ & $6(100)$ & $2(100)$ \\
\hline
\end{tabular}

A prevalência entre sorovares de Salmonella spp. em suínos ou em produtos derivados de carne suína é bastante variável, o que pode ser observado em outros trabalhos realizados na mesma região geográfica deste estudo. BESSA, COSTA e CARDOSO (2004) avaliaram a prevalência de Salmonella spp e de seus sorovares em suínos abatidos em frigoríficos sob inspeção federal no Rio Grande do Sul e verificaram que os sorovares prevalentes foram: Typhimurium (24,3\%), Agona (19,9\%), Derby $(13,2 \%)$ e Bredeney (12\%). MÜRMANN, SANTOS e CARDOSO (2008) avaliaram lingüiça suína frescal comercializada na região de Porto Alegre (RS) e observaram a ocorrência de 12 sorovares, embora Branderburg, Panama, Derby e Typhimurium tenham sido os mais comumente isolados.

Os padrões de resistência aos antibióticos variam entre os sorovares (CARRAMINANA et al., 2004). A suscetibilidade dos isolados de Salmonella Infantis e S. Typhimurium aos 14 antibióticos testados é apresentada na Tabela 3.

A sensibilidade aos antibióticos testados foi dependente do sorovar, conforme descrito por MÜRMANN, SANTOS e CARDOSO (2008) e por CARRAMINANA et al. (2004). Esses últimos autores encontraram maior perfil de resistência em Salmonella Typhimurium quando comparado aos demais, o que também foi evidenciado neste estudo. Todos os isolados pertencentes ao sorovar Typhimurium foram resistentes à tetraciclina, resultado semelhante ao verificado por SALAS et al. (2006) e por CARRAMINANA et al. (2004). Os isolados do sorovar Infantis foram sensíveis à sulfonamida, enquanto aqueles pertencentes aos sorovares Typhimurium, Panama e Derby apresentaram sensibilidade à canamicina. O sorovar Derby apresentou sensibilidade a todos os antibióticos testados, com exceção de tetraciclina (sensibilidade intermediária). Já o sorovar Panama demonstrou padrão de sensibilidade variável entre os antibióticos testados, apresentando resistência à ampicilina, carbenicilina, cloranfenicol e tetraciclina, e sensibilidade à aztreonam, canamicina, cefoxitina, ceftriaxona, ciprofloxacina, e trimetoprina. Não foram encontrados isolados resistentes à aztreonam, canamicina, cefoxitina, ceftriaxona, ciprofloxacina e trimetoprina.

A origem do isolado também pode influenciar sua suscetibilidade aos antimicrobianos. CARRAMINANA et al. (2004) verificaram que $96,2 \%$ dos isolados de Salmonella provenientes de frangos foram resistentes à sulfadiazina (um tipo de sulfonamida) e 53,4\% à neomicina. Atribuíram tal resistência ao seu uso freqüente nas diversas etapas da produção de frangos, ou seja, tratamento dos animais, profilaxia, ou como promotor de crescimento. Da mesma forma, KHAN, MELVIN e DAGDAG (2007) avaliaram a sensibilidade de salmonelas isoladas de produtos de origem vegetal (suco de laranja)à antibióticos. 
Observaram que todos os isolados $(n=11)$ foram sensíveis aos antimicrobianos testados, dentre os quais gentamicina, cloranfenicol, canamicina, ampicilina e ácido nalidíxico. Tal perfil difere bastante daquele encontrado neste experimento e em outras pesquisas que avaliaram salmonelas isoladas de alimentos de origem animal.

\section{TABELA 3 - SUSCETIBILIDADE A 14 ANTIBIÓTICOS ENTRE Salmonella Infantis e S. Typhimurium ISOLADAS DE CORTES DE CARNE SUÍNA COMERCIALIZADOS EM FEIRAS-LIVRES NA CIDADE DE PELOTAS, RS}

\begin{tabular}{|c|c|c|c|c|c|c|c|}
\hline \multirow{3}{*}{ Antibiótico (símbolo) } & \multirow{3}{*}{$\mu \mathrm{g} \cdot$ disco $^{-1}$} & \multicolumn{6}{|c|}{ Salmonella } \\
\hline & & \multicolumn{3}{|c|}{ Infantis (\%) } & \multicolumn{3}{|c|}{ Typhimurium } \\
\hline & & $\mathrm{S}$ & 1 & $\mathrm{R}$ & $\mathrm{S}$ & 1 & $\mathrm{R}$ \\
\hline Ácido Nalidíxico (Nal) & 30 & 100 & 0 & 0 & 0 & 57 & 43 \\
\hline Ampicilina (Amp) & 10 & 93 & 0 & 7 & 29 & 0 & 71 \\
\hline Aztreonam (Atm) & 30 & 100 & 0 & 0 & 100 & 0 & 0 \\
\hline Canamicina $(\mathrm{K})$ & 30 & 86 & 14 & 0 & 100 & 0 & 0 \\
\hline Carbenicilina (Car) & 100 & 86 & 7 & 7 & 29 & 0 & 71 \\
\hline Cefalotina (Cfl) & 30 & 100 & 0 & 0 & 29 & 29 & 42 \\
\hline Cefoxitina (Cfo) & 30 & 100 & 0 & 0 & 100 & 0 & 0 \\
\hline Ceftriaxona (Cro) & 30 & 100 & 0 & 0 & 100 & 0 & 0 \\
\hline Ciprofloxacin (Cip) & 5 & 100 & 0 & 0 & 100 & 0 & 0 \\
\hline Cloranfenicol (Clo) & 30 & 93 & 0 & 7 & 14 & 29 & 57 \\
\hline Gentamicina (Gen) & 10 & 93 & 0 & 7 & 43 & 14 & 43 \\
\hline Sulfonamida (Sul) & 300 & 100 & 0 & 0 & 57 & 0 & 43 \\
\hline Tetraciclina (Tet) & 30 & 43 & 50 & 7 & 0 & 0 & 100 \\
\hline Trimetoprina (Tri) & 5 & 100 & 0 & 0 & 100 & 0 & 0 \\
\hline
\end{tabular}

Os valores indicados para S (sensível), I (intermediário), e R (resistente) representam a porcentagem de 14 isolados de Salmonella Infantis e sete de Salmonella Typhimurium.

Um resultado preocupante evidenciado neste estudo foi que $39,1 \%$ dos isolados apresentaram-se multirresistentes: todos os isolados de Salmonella Typhimurium, um isolado de $S$. Infantis e o isolado de $S$. Panama apresentaram resistência a três ou mais antibióticos. Resultado semelhante foi observado por MÜRMANN, SANTOS e CARDOSO (2008) que avaliando lingüiça frescal suína comercializada em Porto Alegre (RS) verificaram resistência de 85,9\% dos isolados a mais de um antibiótico. Esses autores relatam que a disseminação de salmonelas multirresistentes à antibióticos mediante alimentos de origem animal representa risco adicional à saúde pública.

\section{CONCLUSÃO}

Verificou-se elevada ocorrência de Salmonella spp. em cortes de carne suína comercializados em feiras-livres de Pelotas (RS), que associada ao percentual de isolados multirresistentes (3 ou mais antibióticos) representa risco ao consumidor. Foram encontrados 4 sorovares (Infantis, Typhimurium, Derby e Panama) com sensibilidade distinta à antibióticos de importância em clínica médica.

\section{ABSTRACT}

OCCURRENCE AND ANTIBIOTIC SENSIBILITY PROFILE OF Salmonella spp. ISOLATED FROM PORK MEAT CUTS COMMERCIALIZED IN THE OPEN MARKETS IN PELOTAS, RS (BRAZIL)

The objective of the present study was to evaluate the occurrence of Salmonella spp. in 15 samples of pork meat cuts (T-bone, shank, sausage and ribs) commercialized in open markets of Pelotas (RS, Brazil) and 
verify the prevalent serovars, and test the isolates profile of sensitivity to several antibiotics of importance in medicine (nalidixic acid, ampicillin, aztreonam, kanamycin, carbenicillin, cephalothin, cefoxitin, ceftriaxone, ciprofloxacin, chloramphenicol, gentamicin, sulfonamide, tetracycline and trimetoprina). Twelve samples (80\%) were contaminated by Salmonella enterica, serovars Infantis, Derby, Panama and Typhimurium. All isolates were susceptible to trimetoprin, aztreonam, ciprofloxacin, ceftriaxone and cefoxitin. For the other antibiotics, the pattern of sensitivity varied as serovar. In addition, $39.1 \%$ of isolates showed up to be multiresistant.

KEY-WORDS: MICROBIOLOGICAL CONTAMINATION; ANTIBIOTIC; PORK MEAT; Salmonella; MULTIRESISTANT.

\section{REFERÊNCIAS}

1 APHA. American Public Health Association. Compendium of methods for the microbiological examination of foods. $4^{\text {th }}$ ed. Washington, 2001.

2 BANDEIRA, R.M.; NADVORNY, A.; COSTA, M. CARDOSO, M.R.I. Prevalência de Salmonella sp. em suínos ao abate e em cortes de pernil produzidos no Rio Grande do Sul. In: CONGRESSO BRASILEIRO DE VETERINÁRIOS ESPECIALISTAS EM SUÍNOS, 11. Goiânia, 2003. Anais... Goiânia: Associação Brasileira de Médicos Voluntários Especialistas em Suínos, 2003. v. 1. p. 71-72.

3 BAÚ, A.C.; CARVALHAL, J.B.; ALEIXO, J.A.G. Prevalência de Salmonella em produtos de frangos e ovos de galinha comercializados em Pelotas, RS, Brasil. Ciência Rural, v. 31, n.2, p. 303-307, 2001.

4 BAUER, A.W.; KIRBY, E.M. Antibiotic susceptibility testing by standarized single Disk method. Am. J. Clin. Path., v. 45, n. 4 , p. $493-496,1966$.

5 BERENDS, B. R.; URLINGS, H.A.P.; SNIDJDERS, J.M.A.; Van KNAPEN, F. Identification and quantification of risk factors in animal management and transport regarding Salmonella in pigs. International Journal of Food Microbiology, v. 30, n. 1-2, p. 37-53, 1996.

6 BERSOT, L.S. Cadeia produtiva de suínos e disseminação de Salmonella. Revista do Conselho Federal de Medicina Veterinária, v. 10, n. 31, p. 15-20, 2004.

7 BESSA, M.C.; COSTA, M.; CARDOSO, M. Prevalência de Salmonella sp. em suínos abatidos em frigoríficos sob inspeção federal no Rio Grande do Sul. Pesquisa Veterinária Brasileira, v. 2, n. 24, p. 80-84, 2004.

8 BRASIL. Agência Nacional de Vigilância Sanitária (ANVISA). Aprova o regulamento técnico sobre padrões microbiológicos para alimentos. Resolução $n^{\circ}$ 12, de 02 de Janeiro de 2001. Diário Oficial [da] República Federativa do Brasil, 10 Jan. 2001, seção I, n. 7-E, p. 45-53.

9 CARRAMINANA, J.J.; ROTA, C.; AGUSTIN, I.; HERRERA, A. High prevalence of multiple resistance to antibiotics in Salmonella serovars isolated from a poultry slaughterhouse in Spain. Veterinary Microbiology, v. 104, n. 1-2, p. 133-139, 2004.

10 CASTAGNA, S.M.F.; SCHWARZ, P.; WAGECK, C.C.; CARDOSO, M.R.I. Prevalência de suínos portadores de Salmonella sp. ao abate e contaminação de embutidos tipo frescal. Acta Scientiae Veterinariae, v. 32, n. 2, p. 141 - $147,2004$.

11 COSTALUNGA, S.; TONDO, E. Salmonellosis in Rio Grande do Sul, 1997-1999. Brazilian Journal of Microbiology, v.33, n. 4. p. 342-346, 2002.

12 DUVAL, E.H.; ARAÚJO, M.R.; SILVA, W.P.; LIMA, A.S.; GANDRA, E.A.; NALÉRIO, E.; VON LAER, A.E. Perfil antimicrobiano de Salmonella spp. isolada em linha de processamento de lingüiça suína. In.: CONGRESSO BRASILEIRO DE VETERINÁRIOS ESPECIALISTAS EM SUÍNOS, 11, Goiânia, 2003. Anais... Goiânia: Associação Brasileira de Médicos Veterinários Especialistas em Suínos, 2003. v. 1. p. 69-70.

13 FLUIT, A.C. Towards more virulent and antibiotic-resistant Salmonella? FEMS Immunology and Medical Microbiology, v. 43, n. 1, p. 1-11, 2005.

14 KHAN, A.A.; MELVIN, C.D.; DAGDAG, E.B. Identification and molecular characterization of Salmonella spp. from unpasteurized orange juices and identification of new serotype Salmonella strain S. enterica serovar Tempe. Food Microbiology, v. 24, n. 5, p. 539-543, 2007

15 LIMA, E. S.; PINTO, P.S.A., SANTOS, J. L.; VANETTI, M.C.D.; BEVILACQUA, P.D.; ALMEIDA, L.P.A.; PINTO, M.S.; DIAS, F.S. Isolamento de Salmonella sp e Staphylococcus aureus no processo do abate suíno como subsídio ao sistema de Análise de Perigos e Pontos Críticos de Controle - APPCC. Pesquisa Veterinária Brasileira, v. 24, n. 4, p. 185190, out./dez. 2004.

16 LOGUÉRCIO, A.P.; ALEIXO, J.A.G.; VARGAS, A.C.; COSTA, M.M. ELISA indireto na detecção de Salmonella spp. em lingüiça suína. Ciência Rural, v. 32, n.6, p.1057-1062, 2002. 
17 MÜRMANN, L.; SANTOS, M.C.; CARDOSO, M. Prevalence, genetic characterization and antimicrobial resistence of Salmonella isolated from fresh pork sausages in Porto Alegre, Brazil. Food Control, 2008. In press.

18 NCCLS. National Committee For Clinical Laboratory Standards. Performance standards for antimicrobial disk susceptibility tests. Villanova, PA, 1984.

19 SALAS, C.M.; GIL-SETAS, A.; MAZÓN, A. Etiología y sensibilidad antibiótica de las infecciones extrahospitalarias más frecuentes. Anales del Sistema Sanitario de Navarra, v.29, n. 1, p. 27-36, 2006.

20 SWANENBURG, M.; URLINGS, H.A.P.; SNIJDERS, J.M.A.; KEUZENKAMP, D.A.; KNAPEN, F.V. Salmonella in slaughter pigs: prevalence, serotypes and critical control points during slaughter in two slaughterhouses. International Journal of Food Microbiology, v. 70, n.3, p. 243-254, 2001.

21 WITTE, W. International dissemination of antibiotic resistant strains of bacterial pathogens. Infection, Genetics and Evolution, v. 4, n. 3, p. 187-191, 2004.

22 WHO. World Health Organization WHO global principles for the containment of antimicrobial resistance in animals intended for food. Disponível em: <http://whqlibdoc.who.int/hq/2000/WHO_CDS_CSR_APH_2000.4.pdf>. Acesso em: 15 de outubro de 2008. 